\title{
Estrés e ideación suicida en estudiantes universitarios
}

\author{
López-García Ricardo Natanael*, Martínez-Martínez Edgar Ricardo*, Palacios-Martínez Cinthya*, \\ Rodríguez-de Luna Nallely*, Rodríguez-Romero Dulce Viridiana*, Sánchez-Macías Francisco*, \\ Rodríguez-Nava Víctor Federico**, Rodríguez-Medina María de Lourdes**, González-Flores Silvia \\ Patricia $^{* *}$
}

\begin{tabular}{|c|c|}
\hline 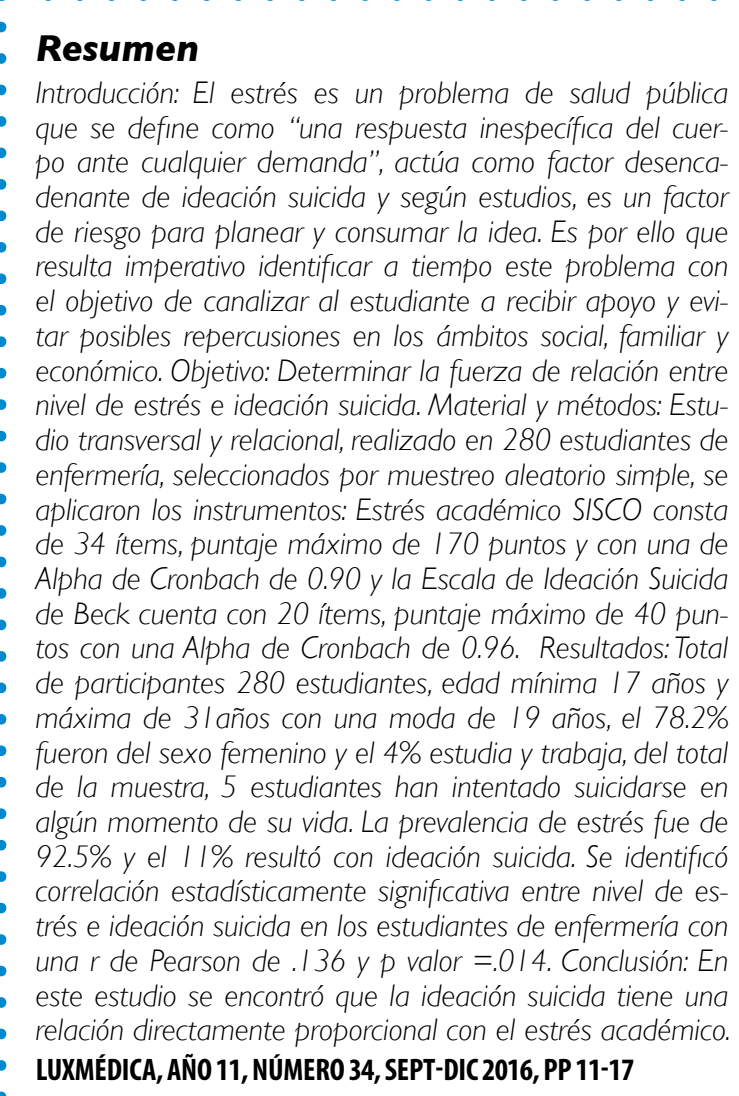 & 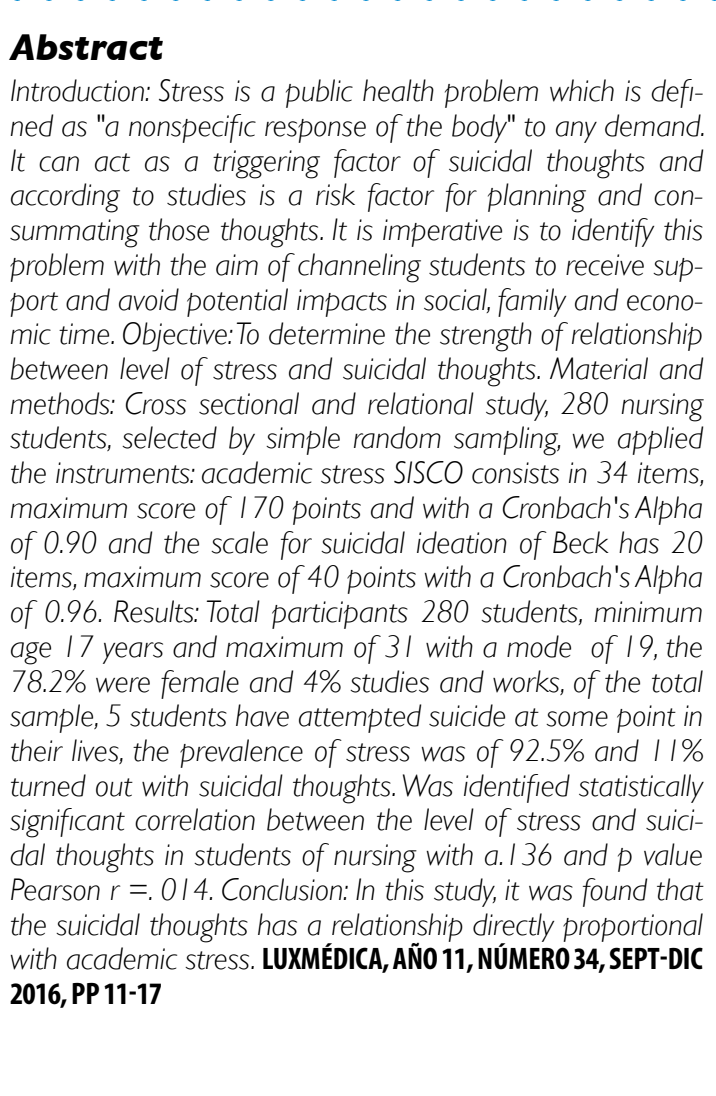 \\
\hline & \\
\hline
\end{tabular}

* Estudiantes de la Licenciatura en Enfermería, Departamento de Enfermería, Centro de Ciencias de la Salud, Universidad Autónoma de Aguascalientes, Aguascalientes, México.

** Profesores investigadores de tiempo completo, Departamento de Enfermería, Centro de Ciencias de la Salud, Universidad Autónoma de Aguascalientes, Aguascalientes, México.

Fecha de recibido: 10 de agosto 2015

Fecha de aceptación: 15 de junio 2016

Correspondencia: MCBE Silvia Patricia González Flores, Departamento de Enfermería del Centro de Ciencias de la Salud de la Universidad Autónoma de Aguascalientes. Avenida Universidad No 940, Edificio 105 Ciudad Universitaria. Código postal. 20131. Teléfono (449) 9108437. Aguascalientes, Ags. México. Correo electrónico: spgonza@correo.uaa.mx 


\section{Introducción}

El estrés es un problema de salud pública y fue acuñado por Hans Selye en 1936, quien lo definió como "la respuesta no específica del organismo a cualquier demanda de cambio". Selye se esforzó sin éxito durante toda su vida para encontrar una definición satisfactoria de estrés. En el intento de extrapolar sus estudios en animales a los seres humanos para que la gente entendiera lo que quería decir, que redefinió el estrés como "la tasa de desgaste en el cuerpo". " Cuando éste se origina en el contexto educativo, se convierte en una manera de adaptarse a las exigencias educativas; algunos investigadores como Dyson y Renk en 2006 y Putwain en 2007 han mencionado que el nivel de estrés aumenta conforme el estudiante progresa en sus estudios y que llega a grados altos cuando cursan sus estudios universitarios. ${ }^{2}$ El estrés se describe con frecuencia como una sensación de agobio, preocupación y agotamiento. El estrés puede afectar a personas de cualquier edad, sexo y circunstancias personales y puede dar lugar a problemas de salud tanto física como psicológica. Por definición, el estrés es cualquier "experiencia emocional molesta que venga acompañada de cambios bioquímicos, fisiológicos y conductuales predecibles." 3 La Organización Mundial de la Salud (OMS) define estrés como "el conjunto de reacciones fisiológicas que prepara el organismo para la acción". ${ }^{4}$

Por otra parte, Émile Durkheim en 1897 definió el suicidio como todo caso de muerte que resulte directa o indirectamente de un acto positivo o negativo realizado por la víctima, a sabiendas del resultado que se producirá. ${ }^{5}$ La ideación suicida se desarrolla lentamente, siendo el último síntoma del episodio mayor, en el que frecuentemente hay pensamientos de muerte y tentativas suicidas. Estas ideas pueden variar desde la creencia de que los demás estarían mejor si uno se muriese hasta los pensamientos pasajeros, pero presentados en forma obsesiva, acerca del hecho de suicidarse, o los auténticos planes específicos sobre cómo cometer el suicidio. Estas ideas, respecto a su frecuencia, intensidad y lealtad, pueden ser muy variables. ${ }^{6}$ Luz de Lourdes Eguiluz define ideación suicida como aquellos pensamientos intrusivos y repetitivos sobre la muerte auto-infringida, sobre las formas deseadas de morir y sobre los objetos, circunstancias y condiciones en que se propone morir.

Por una parte, las investigadoras Medina Mora y González Forteza del Instituto de Salud Mental, señalan que el suicidio puede entenderse como un proceso que comienza con la idea de suicidarse, pasa por la tentativa o los intentos de suicidio, hasta concluir con la muerte auto infringida, es decir, con el suicidio consumado. ${ }^{7}$ Por otra parte, Sergio A. Pérez Barrero menciona que la idea suicida consistente en el pensamiento de acabar con la propia existencia. La ideación suicida surge como salida fácil a la situación o problema que lo aquejan y/o daña psicológicamente. ${ }^{8}$ 
La ideación, se puede concebir como el antecedente de la planeación e intento suicida ${ }^{8}$, es el principal predictor de futuros intentos e incluso del suicidio consumado, está catalogado por la Organización Mundial de la Salud (OMS) como un problema de salud pública a nivel global, y no es posible encontrar cifras estadísticas confiables debido a que existen sub-registros debido a que el problema aparece oculto en la mente del ser de cuidado y no siempre es objetivado. Sin embargo, según la revista Salud Pública de México, en el artículo denominado "Suicidio y conductas suicidas en México: retrospectiva y situación actual" 9 encontró que en México la prevalencia de ideación suicida entre los adultos de 18 a 29 años es del $9.7 \%$ y 3.8\% reportaron intento de suicidio. ${ }^{10}$ En el 2007, entre los habitantes de la República Mexicana, se registró que 6601210 tuvieron ideación suicida en los últimos 12 meses, 593600 personas intentaron suicidarse y 99731 utilizaron servicios médicos como consecuencia de un intento de suicidio. ${ }^{11}$

Con estos antecedentes, consideramos que es importante identificar a tiempo en la población de estudiantes, factores de riesgo como el estrés con la intención de ofrecer el apoyo necesario. Ya se ha mencionado que el estrés actúa como un factor desencadenante de ideación suicida y según estudios, es un factor de riesgo para planear y consumar la idea. ${ }^{12}$ El objetivo de este trabajo fue identificar, en una muestra de estudiantes universitarios, la relación entre el estrés y la ideación suicida.

| | | | | | | | | | | | | | | | | | | | | | | | | | | | | | | | | | | | | | | | | | | | | | | | | | | | | | | | | | | | | | | | | | | | | | | | | | | | | | | | | | | | | | | | | | | | | | | | | | | | | | | | | | | | |

\section{Material y métodos}

Se realizó un estudio prospectivo, observacional, analítico, transversal, relacional. Como criterio de inclusión fue ser estudiante de la Licenciatura en Enfermería de la Universidad Autónoma de Aguascalientes; fueron eliminados los estudiantes que no desearon participar y estudiantes que contestaron los instrumentos de manera incorrecta. El tamaño de la muestra fue de 258 estudiantes, seleccionados por muestreo aleatorio simple, más el índice de corrección, el cálculo del tamaño de la muestra fue de 280 estudiantes, encuestados en el periodo de noviembre a diciembre del 2014 en ambos turnos (matutino y vespertino). Se aplicaron los instrumentos siguientes: "Estrés académico SISCO," que es autoadministrado, consta de 34 ítems, su puntaje máximo es de 170 puntos y el alpha de Cronbach de 0.90; este cuestionario está diseñado a partir de variables relacionadas con el estrés: nivel de estrés auto-percibido, estresores, síntomas y estrategias de afrontamiento evaluadas a través de una escala de Likert; "Escala de Ideación suicida de Beck" mide la prevalencia de la ideación suicida, cuenta con 20 ítems y una alpha de Cronbach de 0.96. Si la puntuación de los ítems " 4 " y " 5 " fuera "1" o "2" se considera que la persona ha tenido una ideación suicida; cuando es " 0 ", se omiten los ítems siguientes. Por lo que no existe una puntuación que determine la presencia de ideación suicida. Posteriormente se creó una base de datos que se analizó con estadística descriptiva. 


\section{Resultados}

Estudio en el que participaron 280 estudiantes de la Licenciatura en Enfermería de la Universidad Autónoma de Aguascalientes, entre un rango de edad de 17 a 31 años, con moda de 19 años. El $78.2 \%$ de la muestra pertenece al sexo femenino; $94 \%$ son solteros, $2.1 \%$ viven en concubinato y $3.9 \%$ son casados; el $4 \%$ estudia y trabaja a la vez.

La religión predominante es católica con un $89.3 \%, 4.6 \%$ cristiano y $6.1 \%$ no son creyentes; $94.3 \%$ no tiene hijos, el $5 \%$ tiene un hijo y $0.7 \%$ tienen dos hijos. El $86.8 \%$ tiene un nivel socioeconómico medio, $12.9 \%$ bajo y $0.4 \%$ nivel alto, como se muestra en la Tabla 1.

\section{Tabla I}

Distribución de las características socio-demográficas de los estudiantes evaluados. $\mathbf{n}=\mathbf{2 8 0}$

\begin{tabular}{|c|c|c|c|}
\hline & & \multicolumn{2}{|c|}{ Sexo } \\
\hline & & Femenino & Masculino \\
\hline \multirow[t]{4}{*}{ Religión } & Católico & 202 & 48 \\
\hline & Cristiano & 11 & 2 \\
\hline & Testigo de Jehová & 0 & 0 \\
\hline & Ateo & 6 & 11 \\
\hline \multirow[t]{5}{*}{ Ocupación } & Estudiante & 217 & 52 \\
\hline & Obrero & 0 & 0 \\
\hline & Empleado & 0 & 0 \\
\hline & Profesionista & 0 & 1 \\
\hline & Estudiante y trabajador & 2 & 8 \\
\hline \multirow[t]{5}{*}{ Estado Civil } & Soltero & 205 & 58 \\
\hline & Concubinato & 4 & 2 \\
\hline & Casado & 10 & 1 \\
\hline & Divorciado & 0 & 0 \\
\hline & Viudo & 0 & 0 \\
\hline \multirow[t]{5}{*}{ Número de Hijos } & 0 & 207 & 57 \\
\hline & 1 & 11 & 3 \\
\hline & 2 & 1 & 1 \\
\hline & 3 & 0 & 0 \\
\hline & Más de 3 & 0 & 0 \\
\hline \multirow[t]{3}{*}{ Nivel Socioeconómico } & Bajo & 27 & 9 \\
\hline & Medio & 191 & 52 \\
\hline & Alto & 1 & 0 \\
\hline \multirow[t]{8}{*}{ Nivel Máximo de Estudios } & $1^{\circ}$ Semestre & 39 & 12 \\
\hline & $2^{\circ}$ Semestre & 25 & 3 \\
\hline & $3^{\circ}$ Semestre & 35 & 12 \\
\hline & $4^{\circ}$ Semestre & 16 & 6 \\
\hline & $5^{\circ}$ Semestre & 37 & 11 \\
\hline & $6^{\circ}$ Semestre & 19 & 6 \\
\hline & $7^{\circ}$ Semestre & 29 & 7 \\
\hline & $8^{\circ}$ Semestre & 19 & 4 \\
\hline
\end{tabular}


Encontramos que la prevalencia de estrés fue de $92.5 \%$ y en el $11 \%$ se identificaron con ideación suicida (figura 1).

\section{Inventario de Estrés Académico (SISCO)}

El 92.5\% del total de la muestra, (259 estudiantes) tuvieron momentos de estrés durante los últimos seis meses, siendo 206 estudiantes del sexo femenino; además 106 mencionaron tener sobrecarga de tarea y trabajos escolares "casi siempre", 93 "algunas veces", 42 "siempre", 16 "rara vez" y 2 "nunca".

El $2.5 \%$ de los estudiantes, dijeron tener sentimientos de depresión y tristeza "siempre", siendo más frecuente "rara vez" con el $38.6 \%$. El $3.6 \%$ presenta ansiedad, angustia o desesperación "siempre" y solamente el $14.6 \%$ "nunca".

\section{Escala de Ideación Suicida de Beck}

El $2.1 \%$ de la muestra no tienen ningún deseo de vivir; $3.6 \%$ tienen poco deseo de vivir, mientras $1.1 \%$ (3 estudiantes) tienen un deseo de moderado a fuerte de morir y el $7.5 \%$ (21 estudiantes) tienen un deseo débil de morir. Solamente un estudiante tiene deseo fuerte de realizar un suicidio activo y 2 estudiantes evitarían los pasos necesarios para salvar o mantener su vida.

De los 30 estudiantes que tuvieron alguna ideación suicida, $0.7 \%$ del $5^{\circ}$ semestre han intentado suicidarse en más de dos ocasiones y $1.1 \%$ del $1^{\circ}, 2^{\circ}$ y $6^{\circ}$ semestre lo han intentado una vez.

Durante el transcurso del semestre los estudiantes que tuvieron momentos de estrés $\mathrm{y} / \mathrm{o}$ ansiedad, 10 de ellos presentaron poco deseo de vivir y 6 ningún deseo de vivir. Mientras 21 manifestaron tener un débil deseo de morir y 3 deseo fuerte de morir.

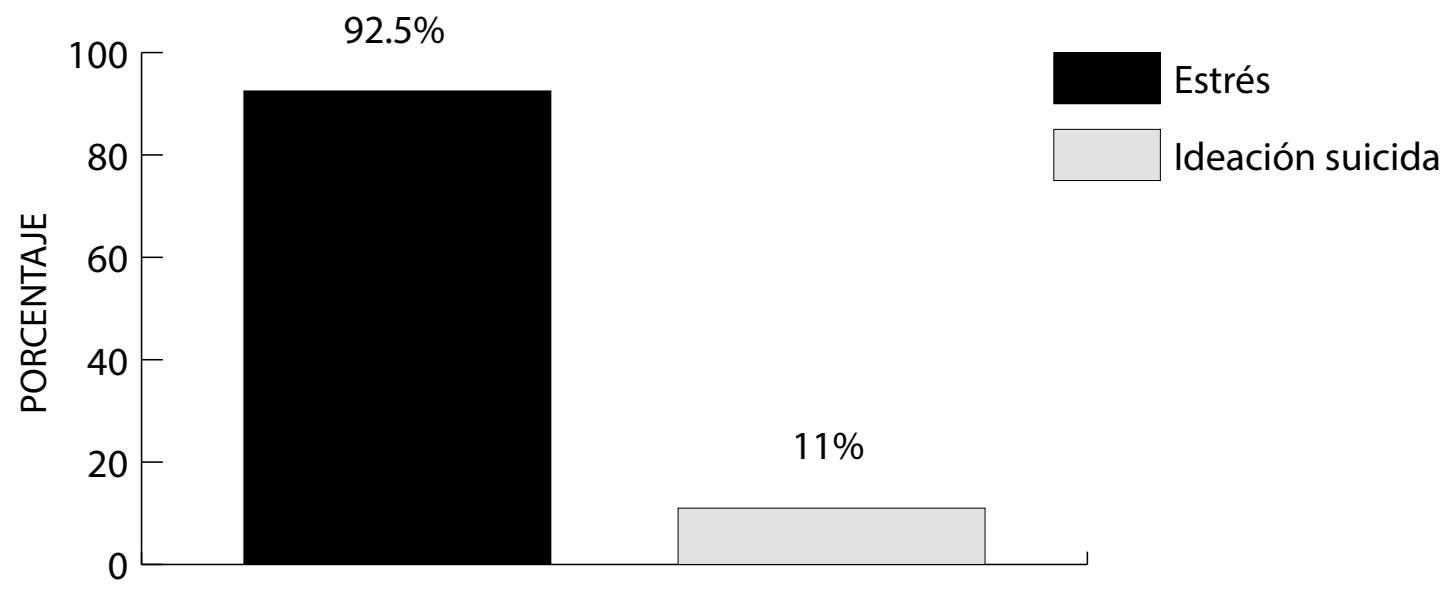

Figura 1. Prevalencia del estrés e ideación suicida Fuente. Instrumento de Estrés Académico SISCO e Instrumento Escala de Ideación Suicida

Se identificó correlación estadísticamente significativa entre nivel de estrés e ideación suicida en los estudiantes de en- fermería con una $r$ de Pearson de .136 y $p$ valor $=.014$. Tabla 2 . 


\title{
Tabla 2
}

\begin{abstract}
Correlación: Estrés e Ideación suicida. Fuente. Instrumento de Estrés Académico SISCO e Ins-
\end{abstract} trumento Escala de Ideación Suicida

\begin{tabular}{|lcc|}
\hline & \multicolumn{3}{c|}{ Correlaciones } \\
\hline Estrés e Ideación Suicida & Sig. (Unilateral) & Correlación de Pearson \\
\hline & .014 & $.136^{*}$ \\
\hline
\end{tabular}

\section{Discusión}

Nuestros resultados muestran que existe una correlación entre la presencia de estrés e ideación suicida, identificados mediante los instrumentos, "Estrés Académico SISCO" y "Escala de Ideación Suicida de Beck", encontramos que entre los estudiantes que manifestaron estrés es más frecuente la ideación suicida. En la carrera de enfermería predomina el sexo femenino, lo cual explica el hallazgo de estrés con mayor frecuencia en este sexo. El $92.5 \%$ del total de la muestra, (280 estudiantes) tuvieron momentos de estrés durante los últimos seis meses, siendo 206 estudiantes del sexo femenino; además 106 mencionaron tener sobrecarga de tarea y trabajos escolares "casi siempre", 93 "algunas veces", 42 "siempre", 16 "rara vez" y 2 "nunca".

Respecto a la ideación suicida se encontraron varios artículos en donde los resultados obtenidos nos hablan de que la mujer es quien más lo piensa, pero el hombre no sólo tiene la idea suicida, sino que es quien lo llega a consumar con mayor fre- cuencia, tal es el caso del estudio que se realizó en Xalapa, Veracruz, en 2005, Estrés, Respuestas de Afrontamiento e Ideación Suicida en Adolescentes ${ }^{13}$, donde los resultados indican que a mayor estrés con la pareja, mayor ideación suicida en los hombres. Mientras que en las mujeres, la ideación suicida se asoció con un mayor estrés social y con la pareja, comparado con nuestro estudio el $10.7 \%$ que presentó ideación suicida, el $8.3 \%$ pertenecen al género femenino, por lo cual las mujeres son más vulnerables y tienden a tener ideación de esta índole más que en relación con los hombres.

En otro estudio, realizado en Lima, Perú, en 2008, "Ideación Suicida y Cohesión Familiar en Estudiantes Preuniversitarios entre 15 y 24 años," 14 los resultados arrojados fueron que el $48 \%$ de los encuestados presentó deseos pasivos de morir, 30\% ideación suicida, $15 \%$ elaboró un plan suicida y $9 \%$ intentó suicidarse. Se identificaron otros factores de riesgo en ambos subgrupos como el antecedente familiar de intento suicida y el hecho de vivir solo.

\section{Conclusiones}

Los resultados de este trabajo nos indican que se debe implementar un programa de detección y manejo del estrés entre los estudiantes universitarios. La identificación de factores adversos psicológico en los estudiantes pueden no sólo repercutir en su desempeño académico, sino también en la disminución de la frecuencia de la ideación suicida. Se requiere continuar con esta línea de investigación, abarcando a mayor número de estudiantes. 


\section{Bibliografía}

1. Cruz Martín C, Vargas Fernández L. ESTRES. Entenderlo es manejarlo. México, D.F.: Alfaomega Grupo Editor, 2001.

2. OMS / Organización Mundial de la Salud. [Online].; 2004 [cited 2013 Marzo 15. Available from: http:// www.who.int/occupational_health/publications/ pwh3sp.pdf.

3.APA J. apa.org. [Online].; 2016 [cited 2016 Abril 15. Available from: http://www.apa.org/centrodeapoyo/estres-cronico.aspx.

4.Pulido Rull MA. UV. [Online].; 2011 [cited 2015 Enero 1. Available from: http://www.uv.mx/psicysalud/ psicysalud-21-1/21-1/Marco-Antonio-Pulido-Rull. pdf.

5. ISSEA. Instituto de Servicios de Salud Del Estado De Aguascalientes. [Online].; 2014 [cited 2015 Enero 1. Available from: http://www.issea.gob.mx/mortgral. asp

6. Leal Zavala, RA, Vásquez Rodríguez, LE. Ideación suicida en adolescestes cajamarquinos prevalencia y factores asociados. [Online].; 2012 [cited 2015 Abril 13. Available from: http://alfepsi.org/attachments/ article/188/Ideaci\% C3\% B3n\% 20suicida \% 20 en $\% 20$ adolescentes $\% 20$ cajamarquinos.pdf.

7. Eguiliz LdL, Córdova MH, Rosales JC. Ante el suicidio Su comprensión y tratamiento. Primera edición ed. Escorza M, editor. México DF: Pax México; 2010.
8. Pérez Barrero Dr. Sergio A. World Psychiatric Association. [Online]. [cited 2016| Abril 9. Available from: http://www.wpanet.org/uploads/Education/ Contributions_from_ELN_Members/la-idea-suicida. pdf.

10. Guilherme Borges, Ricardo Orozco, Corina Benjet - Maria Elena Medina-Mora. Suicidio y conductas suicidas en México: retrospectiva y situación actual. [Online].; 2010 [cited 2015 Abril 13. Available from: http://www.scielo.org.mx/scielo.php?script=sci_art text\&pid $=$ S0036-36342010000400005.

11. Contreras Carlos M, Gutiérrez García Ana G. Bases biológicas del suicidio. [Online].; 2007 [cited 2015 Abril 13. Available from: http://www.uv.mx/cienciahombre/revistae/vol20num1/articulos/suicidio/.

12. Borges G, Orozco R, Benjet C, Medina-Mora ME. Suicidio y conductas suicidas en México: retrospectiva y situación actual. Salud Publica Mex 2010;52:292304 Available from: http://bvs.insp.mx/rsp/articulos/articulo.php?id $=002486$.

13. Serrano-Pereira MG, Flores-Galaz MM. Estrés, respuestas de afrontamiento e ideación suicida en adolescentes. Psicología y Salud,2005;15(2):221-230

14. Muñoz MJ, Pinto MV, Callata CH, Napa DM, Perales CA Ideación suicida y cohesión familiar en estudiantes preuniversitarios entre 15 y 24 años, Lima 2005. Rev Perú Méd Exp Salud Pública 2006; 23(4):239246. Disponible en: http://www.scielo.org.pe/pdf/ rins/v23n4/a02v23n4.pdf. 